\title{
Micromechanical model for rapid prediction of plain weave fabric composite strengths under biaxial tension
}

\author{
Jiangbo Bai' ${ }^{1}$ Zhenzhou Wang ${ }^{2 *}$, Adam Sobey ${ }^{2}$, Ajit Shenoi ${ }^{3}$ \\ $\underline{\text { 1.School of Transportation Science and Engineering, Beihang University, Beijing, People's }}$ \\ Republic of China \\ 2. Fluid Structure Interactions Group, University of Southampton, Southampton, UK (*, \\ corresponding author: Zhenzhou.Wang@ @oton.ac.uk) \\ 3. Southampton Marine and Maritime Institute, University of Southampton, Southampton, \\ $\underline{\mathrm{UK}}$
}

Abstract: The biaxial properties of plain weave fabric composites are important as they are more representative of the performance under complex loading conditions. Experimental determination of these properties is difficult and Finite Element Analysis provides accurate prediction but is computationally expensive and requires skilled users. To provide a simple and rapid prediction of the strength of plain weave fabric composites under biaxial tension a novel micromechanical model is proposed in this paper. To predict the biaxial tensile strengths the minimum total complementary potential energy principle is used on a micromechanical unit cell where the orthogonally interlaced yarns are idealised as curved beams. The new model is verified with a finite element method model on three warp/weft biaxial loading ratios: 1:1, 2:1 $(1: 2)$ and $3: 1(1: 3)$ and uniaxial experimental data. The model is verified on four types of material, ranging in mechanical properties from carbon to glass fibres, and 11 yarn specifications, including five cases compared to experimental results and six cases compared to the FE model, giving a mean error of $9.85 \%$ and a maximum error of $16.74 \%$ compared to experimental results and a mean error of $10.71 \%$ and a maximum error of $14.67 \%$ compared to the FE model, which demonstrates the effectiveness of the model. The standard deviation of prediction errors among the 11 cases is $2.66 \%$, which demonstrates the robustness of the model for a range of applications. The proposed model is able to predict the uniaxial and biaxial tensile strengths without experimental investigations at the fabric and laminate level and only requires the yarn mechanical properties and specifications. 
Keywords: Plain Weave Fabric Composites; Carbon Fibre; Glass Fibre; Biaxial Tensile Strength; Analytical Modelling; Micromechanical Analysis.

\section{Introduction}

Previously the biaxial tensile moduli of plain weave fabric composites have been investigated using the minimum total complementary potential energy principle by Bai et al. [1]. However, the biaxial tensile strength is also a key property and there are few current analytical models to determine it. Therefore, a new method is derived in this paper meaning that damage under complex loading conditions can be represented using this simple approach. The model allows the prediction of the failure locations and the resulting analysis allows the influences of the different biaxial tensile loading ratios on the biaxial tensile strengths to be better understood. Plain weave fabric composites are increasingly used in aerospace structures as they provide a balance between excellent specific strength and stiffness, impact resistance and low cost [2-4]. These structures, such as the wing skin or the aerostat envelope, encounter complex loading conditions during operation but the current literature focuses on improving the accuracy of prediction for the uniaxial tensile properties without considering how multi-axial loading might effect it. Therefore, it is necessary to investigate the strength of plain weave fabric composites under biaxial tension.

Plain weave fabric composites are produced from yarns placed orthogonally to each other which provide compact weaves where each tow in the warp and weft direction alternates between passing over or under the next tow, illustrated in Figure 1; as opposed to twill or satin weaves where a tow may pass over multiple tows in the other direction. In these materials the crimp, or undulation, of the yarns significantly influences the biaxial tensile strengths and so as the range of applications for plain weave fabric composites grows there is an increasing interest in predicting the mechanical properties and the failure mechanisms to be able to optimise these properties. 
The majority of the available experiments for plain weave fabric composites are dominated by tests performed under a single load direction. However, there are a limited number of experiments that investigate the failure mechanisms of these textiles under biaxial tension including polyester woven fabric biaxial behaviour for sails [5,6], membrane woven fabric composites for airship envelopes $[7,8]$ and dry woven fabric reinforcements $[9,10]$. A key issue in these experiments is that the biaxial tensile strengths are not obtained due to the local stress concentrations in the specimens which cause premature failures. In addition none of the studies investigate stiffer composites where the resin is infused into each yarn. For these stiffer materials Bai et al. [1] investigate the biaxial behaviour through experiments but only the biaxial elastic properties are measured and there are no strength measurements. Cai et al. [11] also experimentally investigate the failure mechanisms of stiff plain weave fabric composites under biaxial tension, where the yarns are infused with resin. In these experiments the failure occurs at the transition region between the loading arm and the central thinning area, which indicates that the achieved biaxial tensile strengths are at local stress concentrations, resulting in lower biaxial tensile strengths than the actual values. Due to these stress concentrations, it is hard to obtain accurate biaxial tensile strengths through experiments.

In addition to the experimental work to determine behaviour of plain weave fabric composites, there is an increasing literature modelling these materials. Finite element method (FEM) is a common technique to simulate the failure of plain weave fabric composites. Karkkainen et al. [12] embed a rapid analytical unit cell micromechanical method directly into a finite element analysis to predict the tensile strength of plain weave fabric composites, where the analytical method replaces the homogenisation estimations to accurately and efficiently compute the stiffness matrices. de Carvalho et al. [13] develop a FEM model based on reduced unit cell, which increases the model's precision in predicting the bending response of the twill weave tows under biaxial loading, while maintaining the computational efficiency. The results show 
that the biaxial tensile strength of the twill weave fabric composites vary with the biaxial tensile loadings but the tendency and the reason of the variation is not discussed. Boubaker et al. [1417] propose a multi-scale discrete model to predict the mechanical behaviour of plain weave fabric composites under biaxial tension. This model considers the interactions between the yarns and the effect of this behaviour on the woven structure using a discrete mass-spring model. The contact forces between the yarns are normal to the interlaced surface, which are the same as the interaction forces defined in the current study. However, the model is not able to predict the biaxial tensile strength of plain weave fabric composites. Hivet and Boisse [18] propose an efficient semi-analytical model, which uses a truss model to predict the mechanical behaviour of plain, twill and satin weave fabric reinforcements under biaxial loadings. This model can be used to obtain the stresses on the tensile surfaces, which defines one tension as a function of the two axial strains, within one minute and accurately matches experimentally obtained properties, where the stress-strain plots from the experiments are matched by the predictions. However, the model is not capable of predicting neither tensile modulus nor strength for stiffer plain, twill and satin weave fabric composites under biaxial tension. Cox et al. [19-21] propose an efficient binary model that simplifies the fibre tows as curved truss beams and represents the influences of yarn interactions by using springs to predict the mechanical properties and failure mechanisms of textile composites, where Yang and Cox [22] and Flores et al. [23] utilise the model to simulate the failure of plain weave fabric composites. Even when using the efficient FEM models to predict the strength of the woven fabric composites, such as the binary model for stiff woven fabric composites and the simplified truss type model for woven composite reinforcements, the required computational cost is higher than using an analytical model, where the time to build the geometry, set parameters, create the mesh and calculate the results for each case study is in order of minutes but an analytical model only requires a few milliseconds. Therefore, there are a growing number of analytical methods 
for predicting the strength of plain weave fabric composites; for example Scida et al. [24] and Naik et al. [25] which are both based on the classical thin laminate theory, but there are limited number of biaxial models. It is essential to investigate the mechanical properties and failure mechanisms of plain weave fabric composites under biaxial loadings as they better represent the behaviour of these materials under complex loading conditions. To provide a solution Escárpita [26] proposes a model to calculate the strength under equal biaxial tension based on reducing a given uniaxial strength. However, the model cannot predict the failure location or the strength when the loading ratios are uneven, including uniaxial tension. Chaouachi et al. [27] propose an analytical model based on Hertz contact theory and the minimum potential energy principle to simulate the yarn interactions and predict the biaxial mechanical properties of plain weave fabric composites. The predicted stress-strain plot accurately matches eight experimentally obtained plots but the model is not able to predict the biaxial tensile strengths or failure locations. Therefore, additional improvements are required to accurately and rapidly model plain weave fabric composites under biaxial tension.

There are several undetermined internal forces and moments that need to be solved after the micromechanical analysis of the unit cell. The minimum total complementary potential energy principle states that the solution of the unknown variables is the one that minimises the total complementary potential energy in all possible statically admissible stress fields [28]. The minimum potential energy principle has a similar definition but it is for solving the undetermined internal displacements or strains [28]. According to this definition the undetermined internal forces can be determined by the known external loadings using the minimum total complementary potential energy principle rather than using the minimum potential energy principle. This approach has been used to accurately predict the tensile strengths and moduli of triaxial weave fabric composites $[29,30]$ and the biaxial tensile moduli of plain weave fabric composites [1]. Therefore, this paper proposes an analytical model using 
the same principle. The similar curved beam assumptions of the idealised yarn geometry and the micromechanical analysis of a unit cell remain valid in the proposed model [1]. In order to validate the model, the predictions from the new analytical model are compared with experimental measurements for uniaxial loading cases. Due to the difficulties of measuring biaxial tensile strengths through experiments, the strengths of plain weave fabric composites under biaxial tensile loadings are verified with FEM in ABAQUS. The predicted failure locations and the influences of different biaxial tensile ratios on the biaxial tensile strengths are discussed. The model is verified against four types of materials with a range of different properties, including glass and carbon fibre plain weave fabric composites, to prove the general applicability of the method ${ }^{1}$.

\section{Geometrical representation of plain weave fabric composites}

Plain weave fabrics exhibit a regular, periodical weave pattern. This simplifies the problem as they can be characterised by identical repeated unit cells, where Figure 2 illustrates a unit cell for a plain weave fabric composite laminate; where the coordinate axes $x, y$ and $z$ represent the longitudinal, transverse and through-thickness directions of the tows. Since the axis $y$ is perpendicular to the xoz plane, it is not illustrated in Figure 2. The derivation of the proposed model has the same starting point as the model proposed by Bai et al. [1]. The geometrical assumptions of the tows are inherited from the literature [1,29] for establishing the analytical model through the unit cells. According to the assumptions, the cross-sectional area, $A$, is the summation of the areas of a rectangle and a circle (two semicircles at both ends) and the moment of inertia, $I$, is the summation of moments of inertia of a rectangle and a circle. The undulated neutral axis, $z$, of the biaxial tows are expressed in eq. (1) as,

\footnotetext{
${ }^{1}$ The model has been uploaded for Open Access: https://doi.org/10.5281/zenodo.1476559
} 


$$
z_{n}=\frac{h_{m}}{2} \sin \frac{\pi x}{2 L_{n}}
$$

where the subscript $n$ equals 1 when representing warp tows and 2 when representing weft tows and the subscript $m$ is equal to 2 for $z_{1}$ and 1 for $z_{2}$. The cosine of the off-axial angle for the undulated tows is expressed in eq. (2) as,

$$
\cos \theta_{n}=\sqrt{1+\left(\frac{\pi h_{m}}{4 L_{n}} \cos \frac{\pi x}{2 L_{n}}\right)^{2}},
$$

where the subscript $m$ equals 2 for $\cos \theta_{1}$ and 1 for $\cos \theta_{2}$. The tow fibre volume fraction, $V_{f}$, is expressed in eq. (3) as,

$$
\begin{gathered}
V_{f}=V_{f 0}\left\{( 4 L _ { 1 } L _ { 2 } H ) ^ { - 1 } \left(2 \int_{0}^{L_{1}}\left(\frac{\pi h_{1}^{2}}{4}+h_{1}\left(w_{1}-h_{1}\right)\right) \cos \theta_{1} d x\right.\right. \\
\left.\left.+2 \int_{0}^{L_{2}}\left(\frac{\pi h_{2}^{2}}{4}+h_{2}\left(w_{2}-h_{2}\right)\right) \cos \theta_{2} d x\right)\right\}^{-1},
\end{gathered}
$$

where $H$ is the thickness of the weave fabric ply and $V_{f 0}$ is the fibre volume fraction of the plain weave fabric composite.

The width, w, and thickness, h, of each tow are illustrated in Figure 1. The simplification of the shape of the cross-section of the tow, for example, as a rectangle, rectangle with semicircle at both ends or ellipse, has an influence on the accuracy of the results. However, the cross-section of the tow is usually very flat and a rectangle with semicircles at either end is the most consistent with the actual situation. This results in more accurate calculations and it is therefore adopted in this paper. Half periods of the undulation length, $2 \mathrm{~L}_{1}$ and $2 \mathrm{~L}_{2}$, are illustrated in Figure 2, which can represent the geometry of a tow in a unit cell since one period of a tow is rotational symmetric and a half period of the tow is axial symmetric. In order to avoid complexity, the analytical model assumes that the 
shape of the tow path is fixed as a smooth and continuous sinusoidal curve and does not change as the load is applied.

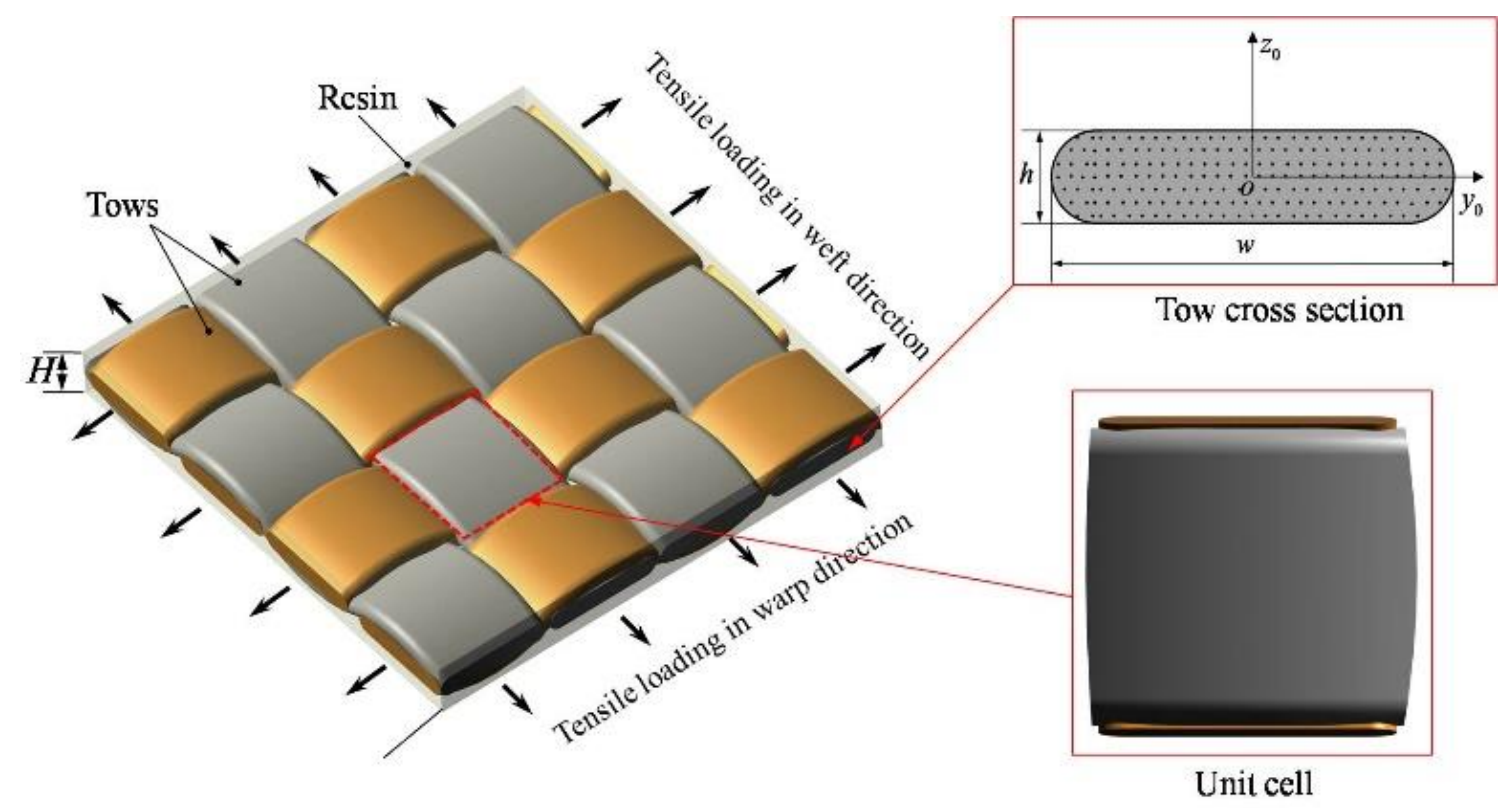

Fig. 1 Illustration of a plain weave fabric composite ply under biaxial tensile loading and the utilised simplification of the yarn cross section.

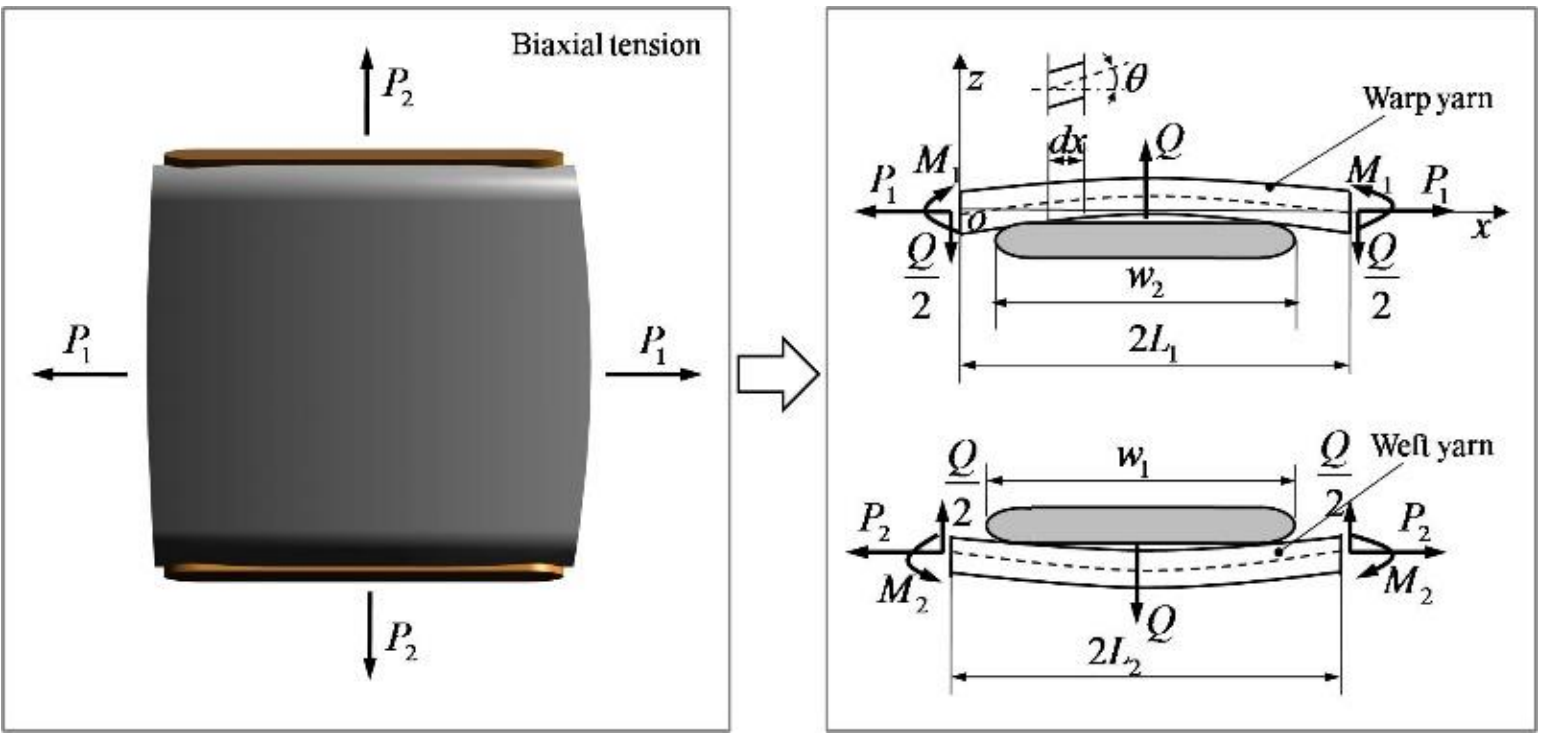

Fig. 2 Illustration of the utilised unit cell with biaxial loading and the assumed internal forces and bending moments on warp and weft yarns (with resin) in this state.

\section{Rapid strength predictions of plain weave fabric composites under biaxial tension}

The ratio between the biaxial tensile loadings, $\lambda$, is defined as $P_{1}$ over $P_{2}$, where $P_{1}$ and $P_{2}$ are the tensile forces along the warp and weft directions respectively, shown in Figure 2. 
According to the micromechanical analysis the internal forces, $F_{n}(x)$, on any cross-section of the warp and weft tows are defined in eq. (4) as,

$$
F_{n}(x)=P_{n} \cos \theta+\frac{Q}{2} \sin \theta
$$

Since it is an in-plane biaxial tension problem, the main interaction between the warp and weft yarns is the normal force at the interlaced section. $Q$ represents this interaction force, which is caused by the yarn interactions under either biaxial or uniaxial tension. The bending moments on the warp and weft tows for each cross-section, $M_{n}(x)$, are expressed in eq. (5),

$$
M_{n}(x)=M_{n}+P_{n} z-\frac{Q}{2} x
$$

where $M_{1}$ and $M_{2}$ are the moments on warp and weft tows induced by tensile loadings. However, the values of $M_{1}, M_{2}$ and $Q$ in eqs. (4) and (5) are unknown. The minimum total complementary potential energy principle is employed to find these undetermined values. The complementary potential energies for the warp and weft tows in the unit cells are calculated in eq. (6),

$$
U_{n}^{*}=\frac{1}{E I_{n}} \int_{0}^{L_{n}} M_{n}^{2}(x) \frac{d x}{\cos \theta_{n}}+\frac{1}{E A_{n}} \int_{0}^{L_{n}} F_{n}^{2}(x) \frac{d x}{\cos \theta_{n}},
$$

where the subscript $m$ equals 2 for $U_{1}^{*}$ and 1 for $U_{2}^{*}$. E is the elastic modulus of a yarn in the longitudinal direction, which is calculated using the rule of mixtures. The complementary potential energies of the warp and weft tows can be obtained by substituting eqs. (1), (2), (4) and (5) into eq. (6). Therefore, the total complementary potential energy of the unit cells, $\Pi^{*}$, is calculated in eq. (7) [1], 


$$
\begin{aligned}
\Pi^{*} & =\frac{1}{E I_{1}} \int_{0}^{L_{1}}\left(M_{1}^{2}+z^{2} P_{1}^{2}+\frac{x^{2}}{4} Q^{2}+2 z M_{1} P_{1}-z x P_{1} Q-x M_{1} Q\right) \frac{d x}{\cos \theta_{1}} \\
& +\frac{1}{E I_{2}} \int_{0}^{L_{2}}\left(M_{2}^{2}+z^{2} P_{2}^{2}+\frac{x^{2}}{4} Q^{2}+2 z M_{2} P_{2}-z x P_{2} Q-x M_{2} Q\right) \frac{d x}{\cos \theta_{2}} \\
& +\frac{1}{E A_{1}} \int_{0}^{L_{1}}\left(\cos ^{2} \theta_{1} P_{1}^{2}+\frac{\sin ^{2} \theta_{1}}{4} Q^{2}+\sin \theta_{1} \cos \theta_{1} P_{1} Q\right) \frac{d x}{\cos \theta_{1}} \\
& +\frac{1}{E A_{2}} \int_{0}^{L_{2}}\left(\cos ^{2} \theta_{2} P_{2}^{2}+\frac{\sin ^{2} \theta_{2}}{4} Q^{2}+\sin \theta_{2} \cos \theta_{2} P_{2} Q\right) \frac{d x}{\cos \theta_{2}} .
\end{aligned}
$$

To minimise the total complementary potential energy for the unit cell, the partial derivatives are taken with respect to $M_{1}, M_{2}$ and $Q$, which is shown in eq. (8) as,

$$
\left[\begin{array}{c}
M_{1} \\
M_{2} \\
Q
\end{array}\right]=P_{1}\left[\begin{array}{ccc}
2 C_{1} & 0 & C_{6} \\
0 & 2 C_{10} & C_{15} \\
C_{6} & C_{15} & 2\left(C_{3}+C_{8}+C_{12}+C_{17}\right)
\end{array}\right]^{-1}\left[\begin{array}{c}
-C_{4} \\
-\frac{C_{13}}{\lambda} \\
-\left(C_{5}+C_{9}+\frac{C_{14}+C_{18}}{\lambda}\right)
\end{array}\right]=P_{1}\left[\begin{array}{c}
D_{1} \\
D_{2} \\
D_{3}
\end{array}\right]
$$

where $C_{j}(j=1,2,3, \ldots, 18)$ are the intermediate variables when solving eq. (7), which are summarised by Bai et al. [1]. The bending moment in the warp direction, $M_{1}$, the bending moment in the weft direction, $M_{2}$, and the internal force, $Q$, are obtained by solving eq. (8) through matrix manipulation, where $D_{i}(i=1,2,3)$ of [1] are the determinants that link the bending moments, $M_{1}$ and $M_{2}$ and the internal force, $Q$, with the external force $P_{1}$. The maximum normal stress on the warp tow is the summation of the axial stress and bending stress [31], which is formulated in eq. (9),

$$
\sigma_{1 \text { max }}=\sigma_{\text {laxial }}+\sigma_{1 \text { bending }}=\frac{P_{1}}{A_{1}}-\frac{M_{1}(x) z_{0}}{I_{1}}
$$


where $z_{0}$ represents the maximum normal stress location, $I_{1}$ is the moment of inertia of warp tows and $M_{1}(x)$ is obtained from eq. (5). According to the micromechanical analysis the maximum normal stress at the trough of the warp tow path, $\sigma_{1 \max }$, is formulated in eq. (10), $z_{0}=-h_{1} / 2$,

$$
\sigma_{1 \max 1}=\frac{P_{1}}{A_{1}}+\frac{h_{1}}{2 I_{1}}\left(\frac{P_{1} h_{2}}{2} \sin \frac{\pi L_{1}}{2 L_{1}}-\frac{Q}{2} L_{1}+M_{1}\right), \quad\left(z_{0}=-h_{1} / 2\right) .
$$

By substituting eq. (8) into the eq. (10), the maximum normal stress at the trough of the warp tow path can be formulated as eq. (11),

$$
\sigma_{1 \max 1}=P_{1}\left(\frac{1}{A_{1}}+\frac{h_{1} h_{2}}{4 I_{1}}-\frac{D_{3} L_{1} h_{1}}{4 I_{1}}+\frac{D_{1} h_{1}}{2 I_{1}}\right), \quad\left(z_{0}=-h_{1} / 2\right)
$$

Using the same process, the maximum normal stress at the crest of the warp tow path, $z_{0}=h_{1} / 2$, is expressed as eq. (12),

$$
\sigma_{1 \max 2}=P_{1}\left(\frac{1}{A_{1}}-\frac{h_{1} h_{2}}{4 I_{1}}+\frac{D_{3} L_{1} h_{1}}{4 I_{1}}-\frac{D_{1} h_{1}}{2 I_{1}}\right), \quad\left(z_{0}=h_{1} / 2\right) .
$$

The maximum normal stresses at the crest and trough of the weft tow path, $\sigma_{2 \max }$, in the unit cells are expressed in eq. (13) for the trough $z_{0}=-h_{2} / 2$,

$$
\sigma_{2 \max 1}=\frac{P_{2}}{A_{2}}+\frac{h_{2}}{2 I_{2}}\left(\frac{P_{2} h_{1}}{2}-\frac{Q}{2} L_{2}+M_{2}\right), \quad\left(z_{0}=-h_{2} / 2\right)
$$

and eq. (14) for the crest $z_{0}=h_{2} / 2$,

$$
\sigma_{2 \max 2}=\frac{P_{2}}{A_{2}}+\frac{h_{2}}{2 I_{2}}\left(-\frac{P_{2} h_{1}}{2}+\frac{Q}{2} L_{2}-M_{2}\right), \quad\left(z_{0}=h_{2} / 2\right),
$$

through a similar derivation process as that used to generate eq. (10) from eq. (9). By substituting eqs. (8) and (12) into eqs. (13)-(14), a similar derivation process as used to 
determine eqs. (11)-(12) can be used to find the maximum normal stress at the trough, $z_{0}=-h_{2} / 2$, expressed in eq. (15),

$$
\sigma_{2 \max 1}=P_{1}\left(\frac{1}{\lambda A_{2}}+\frac{h_{1} h_{2}}{4 \lambda I_{2}}-\frac{D_{3} L_{2} h_{2}}{4 I_{2}}+\frac{D_{2} h_{2}}{2 I_{2}}\right), \quad\left(z_{0}=-h_{2} / 2\right),
$$

and for the stress in the crest, $z_{0}=h_{2} / 2$, as eq. (16),

$$
\sigma_{2 \max 2}=P_{1}\left(\frac{1}{\lambda A_{2}}-\frac{h_{1} h_{2}}{4 \lambda I_{2}}+\frac{D_{3} L_{2} h_{2}}{4 I_{2}}-\frac{D_{2} h_{2}}{2 I_{2}}\right), \quad\left(z_{0}=h_{2} / 2\right)
$$

where the first term $\frac{P_{1}}{\lambda A_{2}}$ is the transformation of $\frac{P_{2}}{A_{2}}$, the second term $\frac{P_{1} h_{1} h_{2}}{4 \lambda I_{2}}$ is the transformation of $\frac{P_{2} h_{1} h_{2}}{4 I_{2}}$ and the last two terms are transformed from $\frac{Q L_{2} h_{2}}{4 I_{2}}$ and $\frac{M_{2} h_{2}}{2 I_{2}}$. The maximum stress failure criterion is used in the model since the failure of the plain weave fabric composites is dominated by the failure of the fibre bundle in the direction of the fibre when under tensile loading. The critical external biaxial tensile forces, $P_{f}$, at each crest and trough location are obtained by the tensile failures of warp and weft tows. The failure force for the trough of the warp tow, subscript 1 , is given in eq. (17),

$$
P_{f 1}=X_{t 0}\left(\frac{1}{A_{1}}+\frac{h_{1} h_{2}}{4 I_{1}}-\frac{D_{3} L_{1} h_{1}}{4 I_{1}}+\frac{D_{1} h_{1}}{2 I_{1}}\right)^{-1}
$$

and the failure force for the crest of the warp tow, subscript 2, is given in eq. (18),

$$
P_{f 2}=X_{t 0}\left(\frac{1}{A_{1}}-\frac{h_{1} h_{2}}{4 I_{1}}+\frac{D_{3} L_{1} h_{1}}{4 I_{1}}-\frac{D_{1} h_{1}}{2 I_{1}}\right)^{-1} .
$$

The failure force for the trough of the weft tow, subscript 3, is given in eq. (19),

$$
P_{f 3}=X_{t 0}\left(\frac{1}{\lambda A_{2}}+\frac{h_{1} h_{2}}{4 \lambda I_{2}}-\frac{D_{3} L_{2} h_{2}}{4 I_{2}}+\frac{D_{2} h_{2}}{2 I_{2}}\right)^{-1},
$$

and the failure force for the crest of the weft tow, subscript 4, is given in eq. (20), 


$$
P_{f 4}=X_{t 0}\left(\frac{1}{\lambda A_{2}}-\frac{h_{1} h_{2}}{4 \lambda I_{2}}+\frac{D_{3} L_{2} h_{2}}{4 I_{2}}-\frac{D_{2} h_{2}}{2 I_{2}}\right)^{-1},
$$

by switching the maximum normal stresses at each location with the tensile force $P_{1}$ in eqs. (11)-(12) and (15)-(16) and replacing the maximum normal stresses in these equations by the tensile strength of the tows where $X_{t 0}$ is the tensile strength of the tows in the longitudinal direction. According to the longitudinal tensile strength of the composites obtained from [32], the tensile strength of the tows is calculated from eq. (21),

$$
X_{t 0}=X_{t} V_{f}+X_{t} E_{f}^{-1} E_{m}\left(1-V_{f}\right)
$$

where $X_{t}$ is the fibre tensile strength in the longitudinal direction, $E_{f}$ is the Young's modulus of the fibre and $E_{m}$ is the Young's modulus of the matrix. The critical external tensile force along the warp direction, $P_{f}$, for the plain weave fabric composite ply can be defined as the lowest failure force from eqs. (17)-(20). Therefore, the biaxial tensile strength of the plain weave fabric composites in the warp loading direction is obtained through the critical force divided by the cross-sectional area and shown in eq. (22),

$$
X_{1 \mathrm{t}}=\frac{P_{f}}{2 L_{2} H}=\frac{\min \left(P_{f 1}, P_{f 2}, P_{f 3}, P_{f 4}\right)}{2 L_{2} H},
$$

where $H$ is the thickness of the weave fabric composite ply. The critical external force along weft direction can be found by dividing $P_{f}$ by $\lambda$. Thus the biaxial tensile strength in the weft direction is shown in eq. (23),

$$
X_{2 \mathrm{t}}=\frac{P_{f}}{2 \lambda L_{1} H}=\frac{\min \left(P_{f 1}, P_{f 2}, P_{f 3}, P_{f 4}\right)}{2 \lambda L_{1} H} .
$$

The analytical model can predict the uniaxial tensile strength of the plain weave fabric composites by setting the biaxial tensile ratio as a large value so that $\lambda$ approaches infinity, $\lambda \rightarrow \infty$ (i.e. $P_{2}=0$ ), in the warp direction and approaches to zero, $\lambda \rightarrow 0$ (i.e. $P_{1}=0$ ), in the 
weft direction. Figure 3 illustrates the derivation process which starts by defining the material properties. The total complementary potential energy, found in eq. (7) can be used to determine the bending moments in the warp and weft directions and the internal forces. The maximum normal stress can then be found using eqs. (9) - (14) and the biaxial tensile strengths are calculated from eqs. (22) and (23).

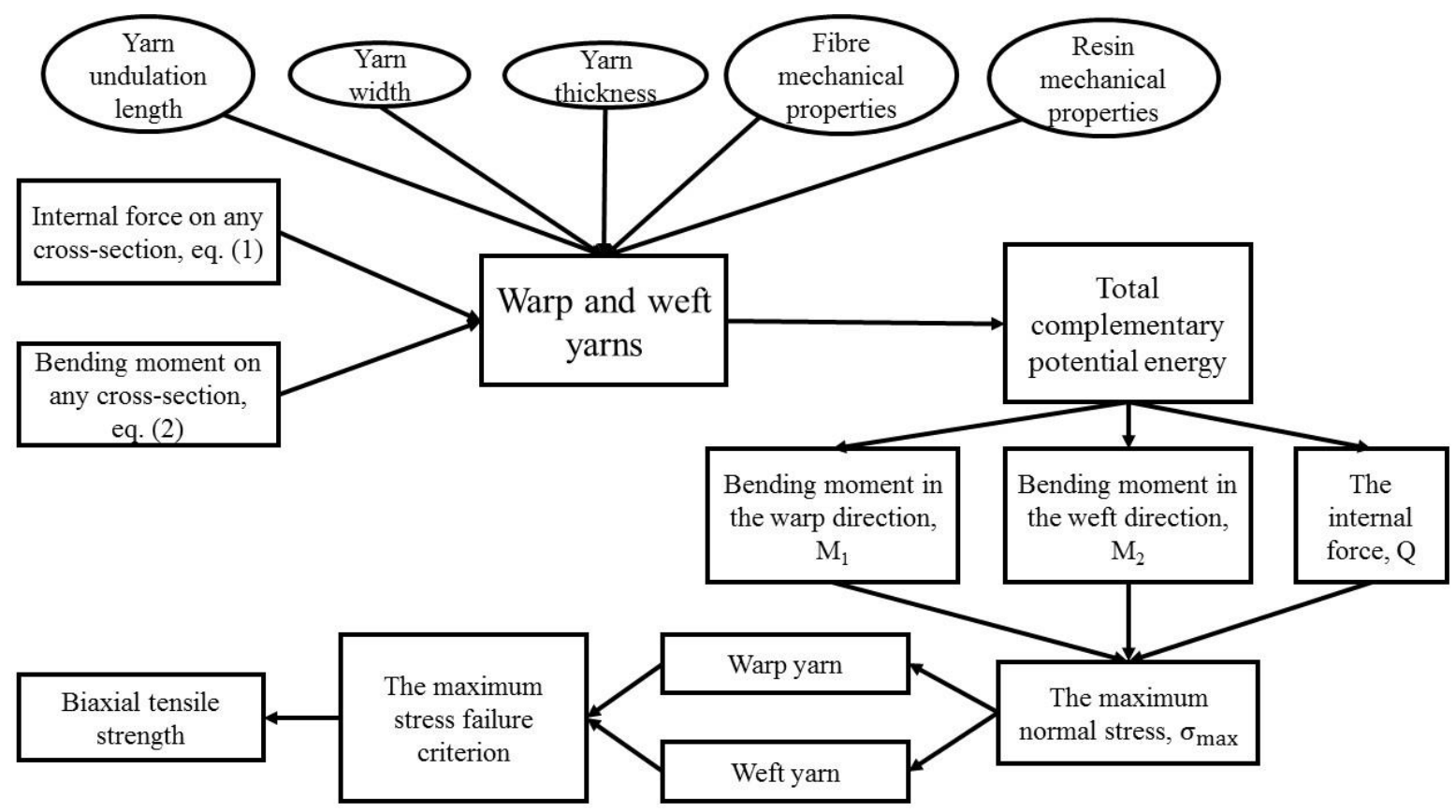

Fig. 3 The process to derive the biaxial strength of a weave fabric using the complementary potential energy principle.

\section{Biaxial verification and uniaxial validation of the analytical model}

In order to validate the analytical model an E-glass/epoxy, EW220/5284, plain weave fabric cruciform composite specimen is designed with 55\% fibre volume fraction using resin transfer moulding assisted by machining and grinding; the geometry and dimensions of the biaxial tensile specimen are shown in Figure 4. The ply thickness of the composite specimens is 0.167 mm with 13 stacked layers. The orientation of the yarn directions for the 13 stacked layers in the specimen were all the same. One quarter of the undulation length, $L$, is $0.714 \mathrm{~mm}$ for the warp and $0.556 \mathrm{~mm}$ for the weft. The width, $w$, and the thickness, $h$, of the tows are $1.0 \mathrm{~mm}$ and $0.080 \mathrm{~mm}$ for the warp direction and $1.2 \mathrm{~mm}$ and $0.067 \mathrm{~mm}$ for weft direction. The fibre 
and the epoxy resin properties are listed in Table 1. The thicknesses of the tow in the warp and weft directions are different in Table 1 . This is because the number of warp and weft tows is not the same in the same width of cloth, resulting in different undulation lengths for the warp and weft tows. In addition, there is a pressure along the thickness direction during the moulding process when it is manufactured, leading to the different widths and thicknesses for the warp and weft tows. The specimen is designed to replicate geometries tested previously in the literature $[1,11]$. The length and width of the cruciform specimens are $130 \mathrm{~mm}$ with a $2.16 \mathrm{~mm}$ thickness for the four loading arms. In order to reduce the influences of the stress concentrations, four elliptical chamfers are designed with a $33.94 \mathrm{~mm}$ length in the major axis and $28.28 \mathrm{~mm}$ length in the minor axis. A diamond-shaped central thinner area is made with three layers of plain weave fabric composite with $0.5 \mathrm{~mm}$ thickness plies. In the specimens, the warp and weft tows are along the $\mathrm{X}$ and $\mathrm{Y}$ directions in Figure 4, respectively. It is difficult to extract the two directional tensile strengths while simultaneously applying biaxial tensile loadings due to the stress concentration at the boundary of the specimens between the loading arm and central area. This is a common problem with no available biaxial data for plain weave fabric composites despite some attempts such as Cai et al. [11] where the biaxial tensile strengths measured were inaccurate due to these stress concentrations. It is common to use experimentally measured mechanical properties of the plain weave fabric composite laminate in a composite laminate FEM model for accurately predicting the biaxial tensile strengths. Since the finite element method is a widely accepted numerical method as well as it is established by the actual specimen and experimentally measured properties, it is used as the benchmark in the verification. Therefore, in order to verify the accuracy and robustness of the model for both uniaxial and biaxial tensile strength predictions, four sets of uniaxial tensile experimental results and the FEM model predictions in ABAQUS with six sets of biaxial loading ratios: 1:1, 2:1 (1:2) and 3:1 (1:3) are used to add confidence to the results. 

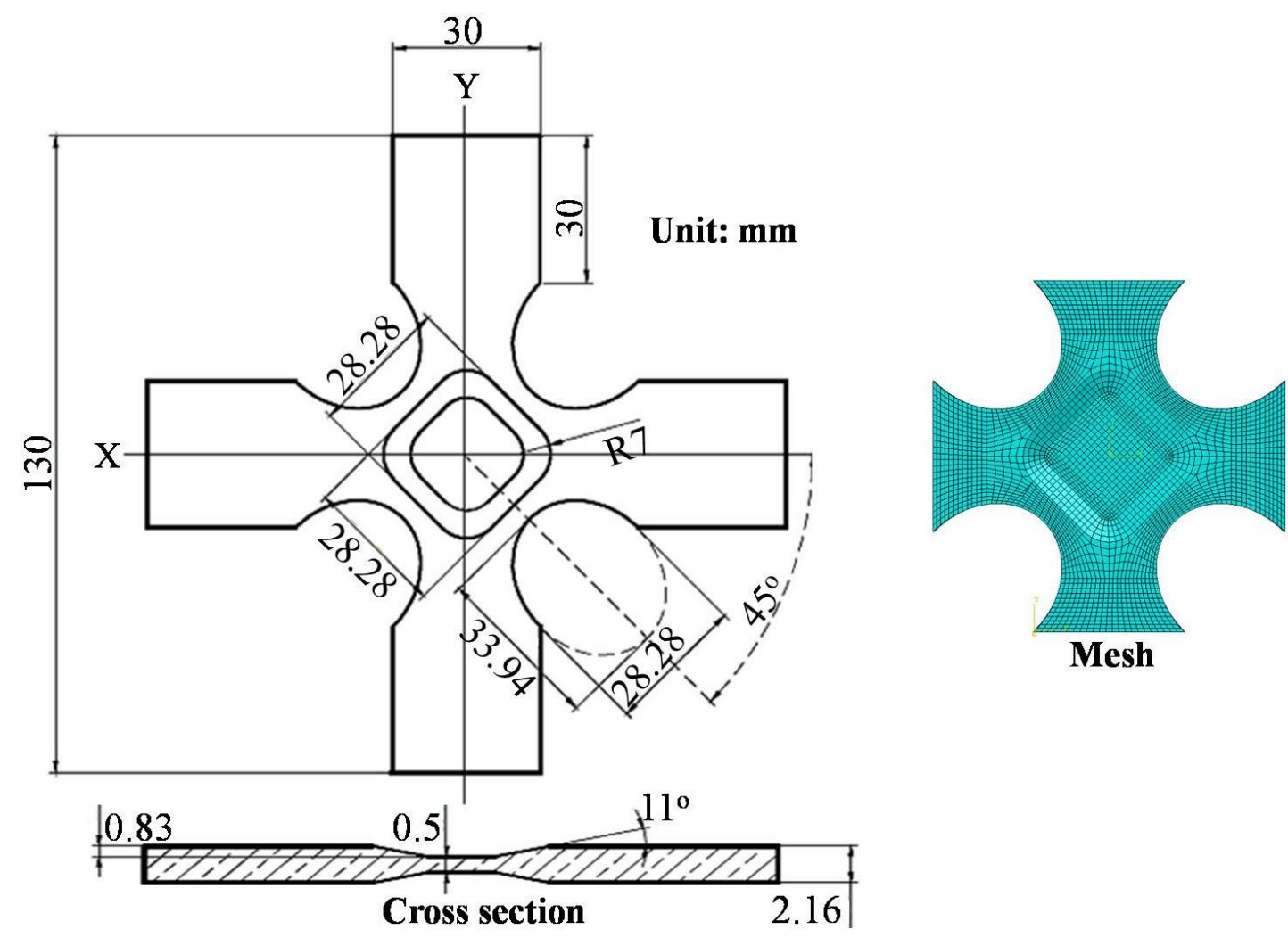

Mesh

Fig. 4 Geometry and dimensions of the biaxial tensile specimen and the finite element mesh generated according to the specimen.

Table 1 Fabric dimensions and mechanical properties of the test specimens

\begin{tabular}{cccc}
\hline Parameters & Values & Parameters & Values \\
\hline$h_{1}(\mathrm{~mm})$ & 0.080 & $w_{2}(\mathrm{~mm})$ & 1.2 \\
$h_{2}(\mathrm{~mm})$ & 0.067 & $H(\mathrm{~mm})$ & 0.167 \\
$L_{1}(\mathrm{~mm})$ & 0.714 & $E_{f}(\mathrm{GPa})$ & 73.0 \\
$L_{2}(\mathrm{~mm})$ & 0.556 & $E_{m}(\mathrm{GPa})$ & 3.4 \\
$w_{1}(\mathrm{~mm})$ & 1.0 & $X_{t}(\mathrm{MPa})$ & 2000 \\
\hline
\end{tabular}

\subsection{Uniaxial tensile tests}

The uniaxial tensile test specimens are manufactured with EW220/5284 plain weave fabric composite using the resin transfer moulding technique and cutting method. The composite preforms are heated at a speed of $5{ }^{\circ} \mathrm{C} / \mathrm{min}$ to $160{ }^{\circ} \mathrm{C}$ and the temperature is held for 1 hour, 
followed by an increase in temperature to $180^{\circ} \mathrm{C}$ with the same heating rate and the temperature is kept static for 2 hours. This process is to reduce defects like voids within the laminates. The plain weave fabric laminates are consolidated under a room-temperature curing process. Finally, the laminates are cut according to ASTM D3039M-2000(R06) to obtain the uniaxial tensile specimens. The total length of each specimen is $250 \mathrm{~mm}$, width is $25 \mathrm{~mm}$, and the thickness is $2 \mathrm{~mm}$. The distance between the grips of the specimen is $100 \mathrm{~mm}$.

The warp and weft direction tensile tests are repeated six times using a MTS880-100Kn servohydraulic machine, meaning there are 12 specimens in total. The static tensile tests are executed at a $3 \mathrm{~mm} / \mathrm{min}$ loading rate, and the load to strain curves are recorded. The stress was calculated at the central part of the specimen. The grips at both ends are held and loaded by the testing machine. The strain and displacements were measured by extensometer with a gauge distance of $25 \mathrm{~mm}$. The straightening of the crimped in-plane tows causes bilinear behaviour in the stress-strain plot in both the uniaxial and biaxial tensile experiments $[1,33]$. However, the bilinear behaviour of glass plain weave fabric composites is different from the elastoplastic behaviour exhibited by metal matrix composites [34] and nanocomposites [35], as the behaviour is caused by an initial hardening phase as the crimped tows straighten rather than a plastic deformation. Therefore, the ultimate tensile strength in the warp and weft directions is measured according to ASTM D3039M-2000(R06). The average tensile strengths are 492.52 MPa in warp direction and 379.57 MPa in weft direction with a standard deviation of 21.61 for the warp direction and 21.49 for the weft direction loadings. The details of the measured tensile strengths in warp and weft directions are listed in Appendix A.

\subsection{Biaxial tensile simulation in ABAQUS}

The biaxial tensile specimens shown in Figure 4 are modelled in ABAQUS, resulting in a model shown in the same figure. The plain weave fabric composite specimen is simplified as 
an anisotropic laminate with 13 plies defined as a solid composite layup in the FEM model. The experimentally measured mechanical properties of the EW220/5284 plain weave fabric composite lamina are used in the FEM model and summarised in Table 2, where the longitudinal and transverse tensile moduli and strengths of the composite were measured according to the ASTM D3039M-2000(R06) and the in-plane shear modulus and strength were measured according to the ASTM standard D3518M-1994(R01). A static analysis is implemented using a linear solver. In order to accurately replicate the boundary conditions in the experiments, the distributed pressure loads are added to the four ends of the cruciform model at the different loading ratios in ABAQUS 6.12 version. Four sets of displacement/rotation boundary conditions are implemented at the ends to constrain the displacements along the loading directions. The yarn undulation lengths in the warp and weft directions are different, $1.428 \mathrm{~mm}$ for the warp tows and $1.112 \mathrm{~mm}$ for the weft tows. Due to the different yarn undulation lengths in the unit cell, the biaxial loadings in the FEM model need to be set differently to those in the analytical model since the biaxial tensile loading ratio in the analytical model represents the warp over weft external forces ratio rather than stress ratio. The biaxial tensile loading ratios in the FEM model are set using the ratio of $P_{1} / 2 L_{2} H$ over $P_{2} / 2 L_{1} H$ to compare against the analytical model, which is $\lambda \frac{L_{1}}{L_{2}}$. Therefore, in order to calibrate the model, the loading ratios set in the FEM model, 3:1, 2:1, 1:1, 1:2 and 1:3, are respectively set as $3.852: 1,2.568: 1,1.284: 1,1.284: 2$ and 1.284:3 for the external biaxial forces in the analytical model. In order to reduce the computational cost while maintaining the accuracy of the FEM model a structured mesh is created using 8-node brick solid elements with reduced integration, C3D8R, and a mesh convergence study is implemented. Seven meshes are compared under $100 \mathrm{MPa}$ biaxial tensile loadings. The stresses in the weft and warp directions and shear stress are plotted to illustrate the convergence, which are shown in Appendices A and B. It is shown that the three stresses converge when the degree of freedom 
reaches 28215 and therefore, it is selected as the final mesh with an illustration in Figure 4. Since the in-plane biaxial tensile loadings are the dominant loadings applied to the finite element model and it assumes there are no defects in the model, the biaxial tensile strength is predicted using Tsai-Hill failure criterion [36],

$$
\frac{\sigma_{1}^{2}}{X^{2}}-\frac{\sigma_{1} \sigma_{2}}{X^{2}}+\frac{\sigma_{2}^{2}}{Y^{2}}+\frac{\tau_{12}^{2}}{S^{2}}=1
$$

where the $\sigma_{1}$ and $\sigma_{2}$ are tensile stresses in longitudinal and transverse directions for the plain weave fabric composite lamina and $\tau_{12}$ is the in-plane shear stress. $X$ and $Y$ are the longitudinal and transverse tensile strengths of the plain weave fabric composite lamina, where the longitudinal strength was obtained from the uniaxial tensile tests in the warp direction and the transverse strength was obtained from the weft direction. $S$ is the in-plane shear strength of the lamina. The in-plane failure is iteratively evaluated, layer by layer on the element with the highest stress at the central thinning area, until the value of Tsai-Hill failure criterion is equal to one, which is the point where the specimen fails. Using this method the biaxial tensile strengths under each loading ratio are obtained.

Table 2 Mechanical properties of the EW220/5284 plain weave fabric composite lamina

\begin{tabular}{cc}
\hline Property & Value \\
\hline Longitudinal elastic modulus $E_{1} / \mathrm{GPa}$ & 14.2 \\
Transverse elastic modulus $E_{2} / \mathrm{GPa}$ & 19.3 \\
Poisson's ratio $v_{12}$ & 0.15 \\
In-plane shear modulus $G_{12} / \mathrm{GPa}$ & 4.3 \\
Longitudinal tensile strength $X_{1} / \mathrm{MPa}$ & 380 \\
Transverse tensile strength $X_{2} / \mathrm{MPa}$ & 493 \\
In-plane shear strength $S_{12} / \mathrm{MPa}$ & 111 \\
\hline
\end{tabular}

\section{Behaviour of plain weave fabrics under uniaxial and biaxial loadings}

\subsection{Verification of analytical method on a range of materials}


In order to investigate the range of applications for the current proposed model another three plain weave fabric composite materials are selected $[37,38]$. Due to the lack of biaxial tensile tests in the literature, only uniaxial tensile strengths are compared. The fibre specifications, material properties and verification results are shown in Table 3. It is shown that the prediction errors for E-Glass/Epoxy, AS4 Carbon/3501-6 Epoxy and TC33 Carbon/Aradur3486 Epoxy plain weave fabric composites are respectively $6.74 \%, 12.20 \%$ and $11.78 \%$. According to the verification results demonstrated above, the proposed analytical model is judged to be valid for predicting the tensile strengths of plain weave fabric composites produced from a range of different materials under uniaxial tension.

Table 3 Experimental and predicted uniaxial tensile strengths with three types of material

\begin{tabular}{|c|c|c|c|c|}
\hline \multicolumn{2}{|c|}{ Material } & E-Glass ${ }^{1}$ & AS4-Carbon ${ }^{2}$ & TC33 Carbon ${ }^{3}$ \\
\hline \multirow{6}{*}{ Yarn Specifications } & $L_{1}(\mathrm{~mm})$ & 0.376 & 0.5 & 0.998 \\
\hline & $L_{2}(\mathrm{~mm})$ & 0.376 & 0.5 & 0.998 \\
\hline & $w_{1}(\mathrm{~mm})$ & 0.732 & 1 & 1.651 \\
\hline & $w_{2}(\mathrm{~mm})$ & 0.732 & 1 & 1.651 \\
\hline & $h_{1}(\mathrm{~mm})$ & 0.1 & 0.04 & 0.1 \\
\hline & $h_{2}(\mathrm{~mm})$ & 0.1 & 0.04 & 0.1 \\
\hline Ply Thickness & $H(\mathrm{~mm})$ & 0.201 & 0.081 & 0.208 \\
\hline Fibre Volume Fraction & $V_{f 0}$ & 0.35 & 0.6 & 0.5 \\
\hline Elastic Modulus of Fibre & $E_{f}(\mathrm{GPa})$ & 72.4 & 221 & 230 \\
\hline Elastic Modulus of Matrix & $E_{m}(\mathrm{GPa})$ & 3.45 & 4.4 & 3.0 \\
\hline $\begin{array}{l}\text { Fibre Tensile Strength in } \\
\text { Longitudinal Direction }\end{array}$ & $X_{t}(\mathrm{MPa})$ & 2413 & 3585 & 3450 \\
\hline \multirow{3}{*}{$\begin{array}{l}\text { Uniaxial Tensile Strengths } \\
\text { Verification (Warp }= \\
\text { Weft) }\end{array}$} & $\begin{array}{c}\text { Experiments } \\
(\mathrm{MPa}) \\
\end{array}$ & 353.27 & 737.5 & 588.43 \\
\hline & Predictions (MPa) & 329.45 & 827.46 & 657.76 \\
\hline & Errors (\%) & 6.74 & 12.20 & 11.78 \\
\hline
\end{tabular}

1. E-Glass/Epoxy [37]

2. AS4-Carbon/3501-6 Epoxy [37]

3. TC33 Carbon/Aradur3486 Epoxy [38] 


\subsection{Effect of loading ratio}

Predicted results from the proposed analytical model are compared with the biaxial predictions from the FEM model and the uniaxial measurements from experiments, summarised in Table 4. The tensile strengths of the plain weave fabric composites under biaxial loading ratios 1:1, 2:1 (1:2), 3:1 (1:3) and $\infty: 1(1: \infty)$ are evaluated through eqs. (22) and (23). Over these ratios it is demonstrated that the maximum error between the warp biaxial tensile strength prediction and the FEM prediction is $11.18 \%$. The warp uniaxial tensile strength prediction shows a similar level of accuracy as the three uniaxial strength verification cases in the previous section, which has an error of $11.64 \%$. For the weft direction the maximum error is $14.67 \%$ between the predictions from the FEM model and the analytical model. The error between the analytical model prediction under weft uniaxial tension and the average of experimental measurements is $16.74 \%$. The mean errors are $10.51 \%$ for warp direction predictions and $12.65 \%$ for weft direction predictions. Figure 5 shows the comparison between the experimental and predicted results for the different loading ratios, illustrating the predicted results accurately match with the experimental measurements and FEM predictions. The differences between the analytical predictions and the experimental and FEM predicted results are caused by the assumptions: that the yarns retain a non-deformable smooth and continuous sinusoidal path, even after loading, and that the cross-section is represented as a flat rectangle with a semicircle at each end. Although a simple basis transform rule can be used for the calculation of biaxial tensile strength, it requires the macroscopic material parameters, such as the fabric and lamina anisotropic moduli and strengths. The proposed model is able to predict the uniaxial and biaxial tensile strengths without experimental investigations at the fabric and laminate level and only requires the yarn mechanical properties and specifications. Although the manufacturer provides the yarn and resin mechanical properties and specifications, it is recommended to remeasure these parameters in order to ensure the reliability of the data. 
Table 4 Experimental and predicted warp and weft biaxial tensile strengths for EW220/5284 plain weave fabric composite

\begin{tabular}{|c|c|c|c|c|c|}
\hline & & $\lambda=1$ & $\lambda=2$ & $\lambda=3$ & $\lambda \rightarrow \infty$ \\
\hline \multirow{8}{*}{$\begin{array}{l}\text { Warp strengths } \\
\qquad X_{1 \mathrm{t}}(\mathrm{MPa})\end{array}$} & FE predictions & $409.77^{\text {FEM }}$ & $556.13^{\text {FEM }}$ & $499.16^{\text {FEM }}$ & $492.52^{\operatorname{Exp}}$ \\
\hline & \&Experiments & & & & \\
\hline & Theoretical & 374.75 & 493.93 & 552.51 & 549.88 \\
\hline & Predictions & & & & \\
\hline & Errors & $8.55 \%$ & $11.18 \%$ & $10.68 \%$ & $11.64 \%$ \\
\hline & Critical & warp yarn, & warp yarn, & warp yarn, & warp yarn, \\
\hline & locations* & $z_{0}=h_{1} / 2$ & $z_{0}=h_{1} / 2$ & $z_{0}=h_{1} / 2$ & $z_{0}=-h_{1} / 2$ \\
\hline & & $\lambda=1$ & $\lambda=1 / 2$ & $\lambda=1 / 3$ & $\lambda \rightarrow 0$ \\
\hline \multirow{8}{*}{$\begin{array}{l}\text { Weft strengths } \\
\qquad X_{2 \mathrm{t}}(\mathrm{MPa})\end{array}$} & FE predictions & & & & \\
\hline & & $264.57^{F E M}$ & $461.35^{F E M}$ & $406.21^{F E M}$ & $379.57^{\operatorname{Exp}}$ \\
\hline & \&Experiments & & & & \\
\hline & Theoretical & 291.82 & 393.67 & 370.07 & 312.60 \\
\hline & Predictions & & & & \\
\hline & Errors & $10.30 \%$ & $14.67 \%$ & $8.90 \%$ & $16.74 \%$ \\
\hline & Critical & warp yarn & warp yarn & weft yarn & weft yarn \\
\hline & locations* & $z_{0}=h_{1} / 2$ & $z_{0}=h_{1} / 2$ & $z_{0}=-h_{2} / 2$ & $z_{0}=-h_{2} / 2$ \\
\hline
\end{tabular}

*See Figures 1 and 2 for details of cross-section at the path peaks and valleys of yarns FEM the value is obtained from Finite Element Method

Exp the value is obtained from experiments 


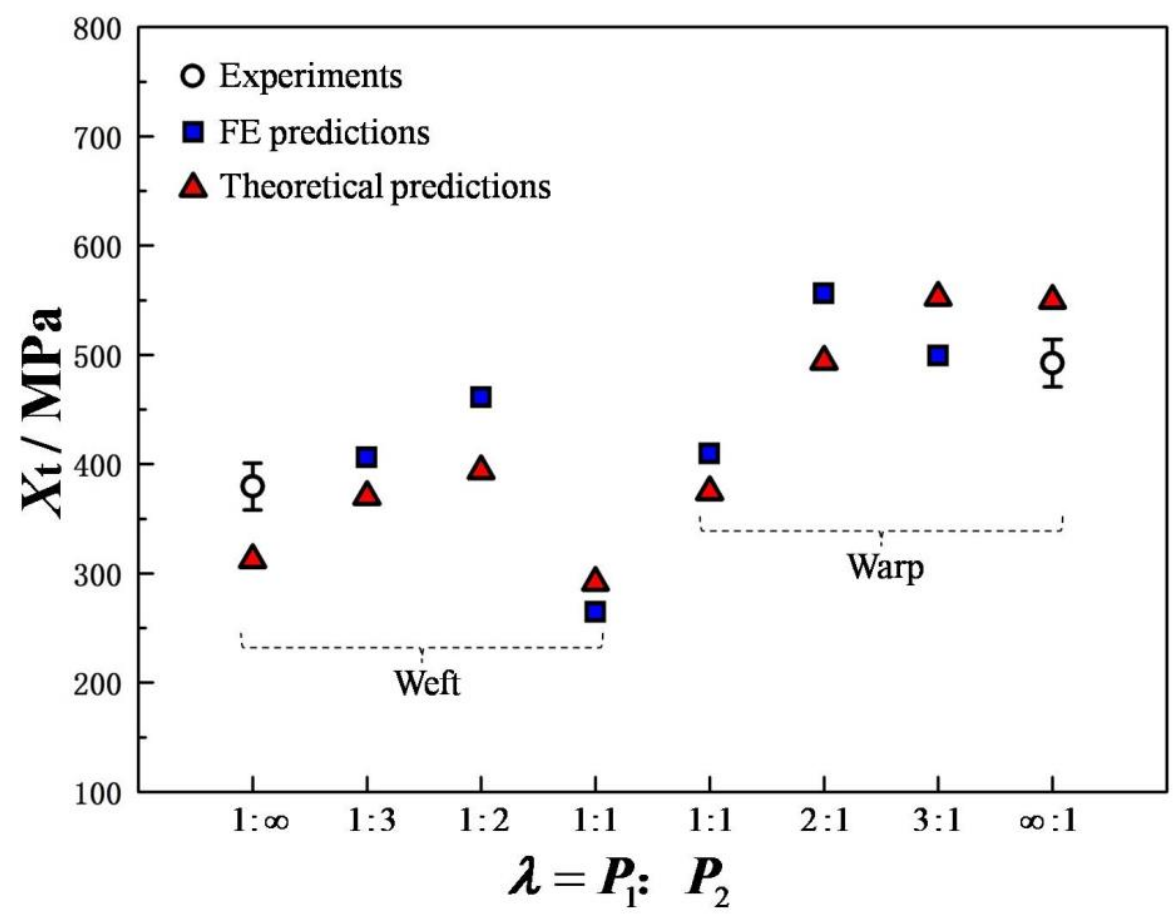

Fig. 5 Verification of the tensile strength predictions under different biaxial tensile force ratios: comparison between the unidirectional loaded experimental data (white circles), FE predictions (blue squares) and analytical predictions (red triangles) for the selected EW220/5284 plain weave fabric composite, where 1:œ and $\infty: 1$ represent unidirectional loading.

\section{Discussion}

The critical locations for the weave fabric are demonstrated for each biaxial loading ratio, shown in Table 4. Although the predicted failure location is not verified with experimental observation, since the failure is instantaneous and it is difficult to observe the micro-failures through the surface of the specimens, the predicted failure locations help to gain insights into the failure mechanisms of plain weave fabric composites. The damage occurs at the peaks of the warp yarn when $\lambda=1,2$ and 3 but at the troughs of the warp yarn when $\lambda \rightarrow \infty$. For the weft yarns, the damage occurs at the peaks of the warp yarns when $\lambda=1$ and $\lambda=1 / 2$. The damage occurs at the troughs of the weft yarns when $\lambda=1 / 3$ and $1 / \infty$. It is found that the failure starts at the warp yarn under biaxial tension until the weft direction loading is three times higher than the warp direction loading. According to the analysis of the fabric 
specifications provided by the manufacturer, the reason is the warp yarns are thicker with longer undulation lengths resulting in higher bending stresses due to a higher internal force, $Q$, and external force, $P$, which induces the moments, $\frac{Q x}{2}$ and $P z$, in eq. (5). The strengths in both the warp and weft direction initially increase and then decrease from $\lambda=1$ to $\lambda \rightarrow \infty$ in the warp direction and from $\lambda=1$ to $1 / \infty$ in the weft direction; this is shown in Table 4 and Figure 5 and applies to both the FEM and analytical models. This phenomenon is caused by the variation in the total stresses caused by the internal bending moments and change of the failure location. When the loading changes from equal biaxial tension to warp uniaxial tension, $\lambda$ from $1: 1$ to $\infty: 1$, or from equal biaxial tension to weft uniaxial tension, $\lambda$ from $1: 1$ to $1: \infty$, the failure location changes from the crest of the tows to the trough of the tows in both directions. The change in stress is caused by the internal force induced bending moment which is increased to balance the increased loading in the warp or weft direction. Since the stress caused by the internal force induced bending moment, $\frac{Q x}{2}$, is opposite to the stresses caused by the tension induced bending moment, $M$, and the tensile forces induced bending moment, $P z$, the total stresses caused by the internal bending moments are reduced; increasing the strength of the material. When the warp direction loading is three times larger than the weft direction loading or the weft direction loading is two times larger than the warp direction loading, the total stress is dominated by the stresses caused by the tensile forces. The increased tensile forces result in a higher total stress, and therefore the strength is reduced. These relationships are reflected in the eqs. (10)-(11) and (14)-(15). Furthermore, the total stresses under uniaxial tension are lower than under equal biaxial tension since the contribution of the stresses caused by the tension induced bending moment, $M$, and the internal force induced bending moment, $\frac{Q x}{2}$, are both significantly lower under uniaxial tension. Therefore, the strength under uniaxial tension is higher than that under equal biaxial tension. This phenomenon is also demonstrated 
by Escárpita [26] where the strength under equal biaxial tensions is calculated by reducing the uniaxial strength.

The fabric specification has a substantial impact on the strength of the plain weave fabric composites, but this is also dependent on the type of load it will see in service. This means there is the potential to optimise the weave fabric to improve its properties but this can be further improved if its operation loads are known, for example a composite under uniaxial loading may differ in weave pattern from that expecting a more complex loading scenario. The model provided here gives a simple and rapid analysis to determine this specification.

Twill and satin weave fabrics are also common in composite applications, since they can be used to produce structures with curved geometries due to their lower crimp. The current model is developed to accurately predict the biaxial tensile strengths of plain weave fabric composites. However, initial feasibility studies show that the model can be extended to predict the biaxial tensile strengths of twill and satin weave fabric composites. To develop these models, the same steps can be followed, where the first step is to obtain the micromechanical behaviour of the satin and twill weave fabric composite unit cells. Then the total complementary potential energy can be calculated and minimised, so that all of the undetermined internal forces and moments can be obtained. Finally, the biaxial tensile strength can be derived.

The influence of the variation of the yarn's modulus on the tensile strength on the plain weave fabric composite is investigated through a parametric study. The tensile strength along the warp and weft directions are investigated for E-glass fibre modulus values from $60 \mathrm{GPa}$ to $90 \mathrm{GPa}$, which is considered to be the feasible range for most commercially available E-glass fibres [39]. This change in fibre modulus results in a yarn modulus variation from $44.47 \mathrm{GPa}$ to 66.23 GPa. It is found that this change in modulus results in a slight reduction in tensile strength from 539.63 MPa to 535.85 $\mathrm{MPa}$ in the warp direction and from $333.49 \mathrm{MPa}$ to $331.16 \mathrm{MPa}$ in the 
weft direction, which is shown in Figure 6. Therefore, the tensile strengths of plain weave fabric composites are not significantly influenced by the variation in the fibre modulus.

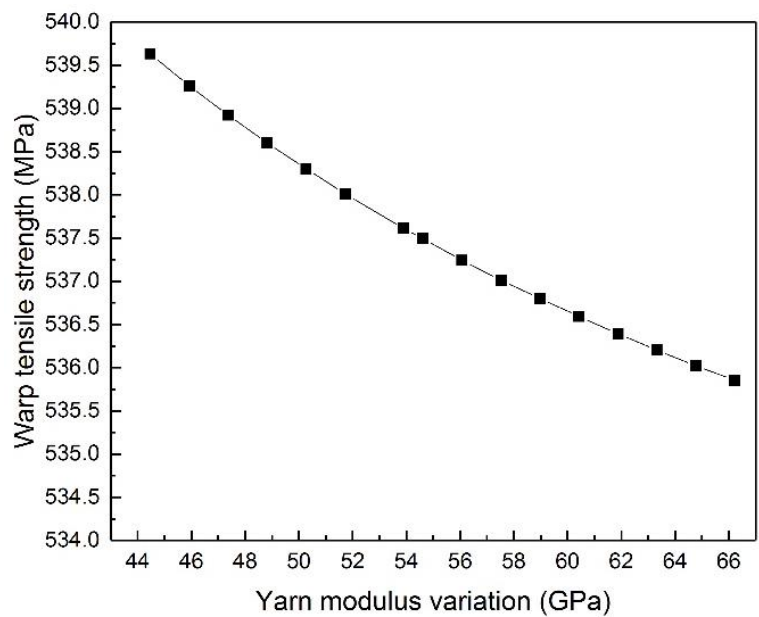

(A)

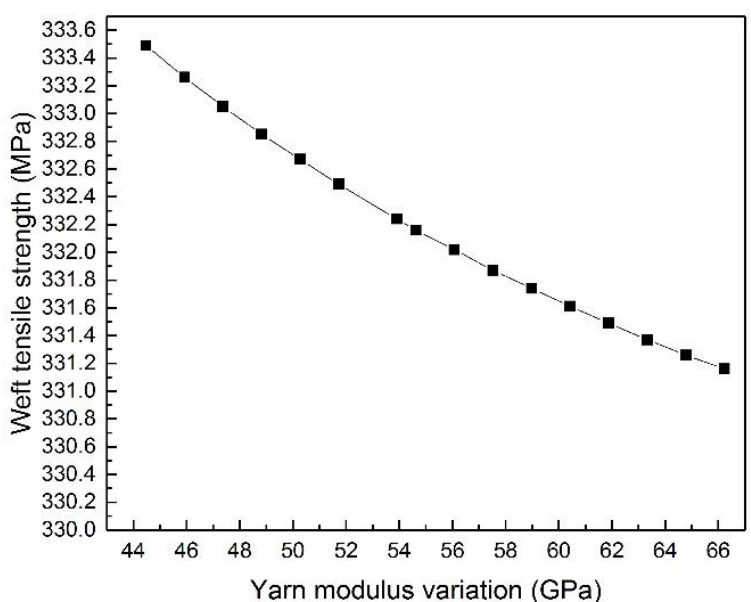

(B)

Fig. 6 The influence of the yarn's modulus variation on the tensile strength of plain weave fabric composites

A limitation of the current research is the difficulty in obtaining the biaxial tensile strength through experiments, due to the inevitable stress concentrations at the transition regions between the loading arms and the central thinning area of the specimens, and therefore the proposed model is verified with the FEM model. While the FEM provides confidence in the accuracy of the method, the ability to determine experimental data will increase the confidence in the approach.

\section{Conclusions}

A novel analytical model is proposed for rapid prediction of the biaxial tensile strengths of plain weave fabric composites. The model is derived based on the minimum total complementary potential energy principle with micromechanical analysis of orthogonally interlaced yarns. The proposed model is verified against the FEM under different biaxial loading cases and validated by experimental measurements under uniaxial tensile loading. Different biaxial tensile loading ratios and various types of materials, ranging in material properties from carbon to glass fibres, are used to show the general applicability of the method. 
By analysing the predicted results, it is demonstrated that the biaxial tensile strengths can be determined from the fibre specifications and biaxial loading ratios. The result is a model giving a mean error of $10.71 \%$ and a maximum error of $14.67 \%$ over the six biaxial tension cases and a mean error of $9.85 \%$ and a maximum error of $16.74 \%$ over five uniaxial tension cases and four types of materials, which indicates the accuracy of the proposed model. Furthermore, the standard deviation of prediction errors among the total 11 cases is 2.66 , which demonstrates the robustness of the model for a range of applications.

\section{Acknowledgements}

This project was supported by the National Natural Science Foundation of China (Grant No. 51875026), the Lloyds Register Foundation and China Scholarship Council.

\section{Data availability}

The raw code of the model required to reproduce these findings are available to download from https://doi.org/10.5281/zenodo.1476559. The processed data required to reproduce these findings are available in Tables 1, 3 and 4 of this manuscript.

\section{Appendix A. Tables of uniaxial tensile tests data and FE model mesh convergence study}

Table A.1 Experimental uniaxial tensile strengths of EW220/5284 PWF composites

\begin{tabular}{ccc}
\hline \multirow{2}{*}{ Specimen No. } & Warp (i.e. $\lambda=\infty)$ & Weft (i.e. $\lambda=0)$ \\
\cline { 2 - 3 } & Strengths $(\mathrm{MPa})$ & Strengths $(\mathrm{MPa})$ \\
\hline 1 & 479.79 & 384.44 \\
2 & 480.47 & 345.23 \\
4 & 536.10 & 366.70 \\
5 & 486.11 & 387.64 \\
6 & 488.69 & 384.67 \\
Average & 483.98 & 408.73 \\
Standard deviation & 492.52 & 379.57 \\
\end{tabular}


Table A.2 Statistics of mesh in mesh convergence study

\begin{tabular}{ccccc}
\hline Mesh & No. of nodes & No. of elements & DOF & CPU time (s) \\
\hline 1 (Coarse) & 2496 & 1526 & 7488 & 7.19 \\
2 & 3195 & 1928 & 9585 & 8.05 \\
3 & 4188 & 2610 & 12564 & 11.97 \\
4 & 5007 & 3136 & 15021 & 13.67 \\
5 & 6945 & 4392 & 20835 & 18.90 \\
6 & 9405 & 5984 & 28215 & 21.60 \\
7 (Fine) & 19120 & 13806 & 57360 & 58.18 \\
\hline
\end{tabular}

\section{Appendix B. The figure of mesh convergence}

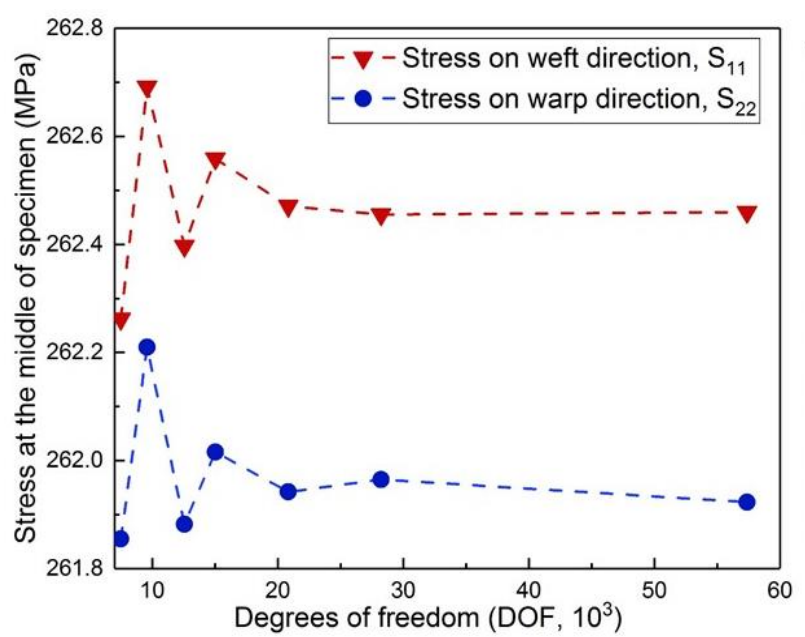

(a)

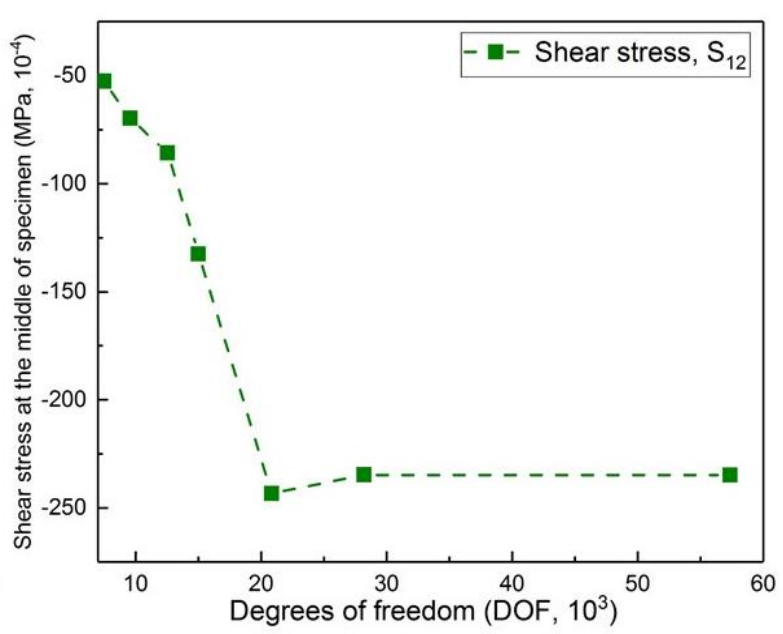

(b)

Fig. B.1 Mesh convergence study: (a) stresses in warp and weft directions; (b) shear stress

\section{References}

[1] Bai JB, Xiong JJ, Shenoi RA, Wang Q. A micromechanical model for predicting biaxial tensile moduli of plain weave fabric composites. J Strain Anal Eng Des 2017;52:333-43. doi:10.1177/0309324717707858.

[2] Xiong JJ, Shenoi RA. General aspects on structural integrity. Chinese J Aeronaut 2019;32:114-32. doi:10.1016/j.cja.2018.07.018.

[3] Bai J, Xiong J, Cheng X. Tear Resistance of orthogonal Kevlar-PWF-reinforced TPU film. Chinese J Aeronaut 2011;24:113-8. doi:10.1016/S1000-9361(11)60014-9.

[4] Xiong JJ, Shenoi RA, Cheng X. A modified micromechanical curved beam analytical model to predict the tension modulus of 2D plain weave fabric composites. Compos Part B Eng 2009;40:776-83. doi:10.1016/j.compositesb.2009.06.004.

[5] Quaglini V, Corazza C, Poggi C. Experimental characterization of orthotropic technical textiles under uniaxial and biaxial loading. Compos Part A Appl Sci Manuf 2008;39:1331-42. doi:10.1016/j.compositesa.2007.07.008.

[6] Ambroziak A, Kłosowski P. Polyester sail technical woven fabric behaviour under uniaxial and biaxial tensile tests. J Theor Appl Mech 2018:227. doi:10.15632/jtam-pl.56.1.227.

[7] Qiu Z, Chen W, Gao C, Hu Y. Experimental and numerical study on nonlinear mechanical properties of laminated woven fabrics. Constr Build Mater 2018;164:672-81.

doi:10.1016/j.conbuildmat.2018.01.004. 
[8] Shi T, Chen W, Gao C, Hu J, Zhao B, Wang P, et al. Biaxial strength determination of woven fabric composite for airship structural envelope based on novel specimens. Compos Struct 2018;184:1126-36. doi:10.1016/j.compstruct.2017.10.067.

[9] Gasser A, Boisse P, Hanklar S. Mechanical behaviour of dry fabric reinforcements. 3D simulations versus biaxial tests. Comput Mater Sci 2000;17:7-20. doi:10.1016/S09270256(99)00086-5.

[10] Boisse P, Gasser A, Hivet G. Analyses of fabric tensile behaviour: Determination of the biaxial tension-strain surfaces and their use in forming simulations. Compos - Part A Appl Sci Manuf 2001;32:1395-414. doi:10.1016/S1359-835X(01)00039-2.

[11] Cai D, Tang J, Zhou G, Wang X, Li C, Silberschmidt V V. Failure analysis of plain woven glass/epoxy laminates: Comparison of off-axis and biaxial tension loadings. Polym Test 2017;60:307-20. doi:10.1016/j.polymertesting.2017.04.010.

[12] Karkkainen RL, Sankar B V. A direct micromechanics method for analysis of failure initiation of plain weave textile composites. Compos Sci Technol 2006;66:137-50.

doi:10.1016/j.compscitech.2005.05.018.

[13] De Carvalho N V., Pinho ST, Robinson P. Numerical modelling of woven composites: Biaxial loading. Compos Part A Appl Sci Manuf 2012;43:1326-37.

doi:10.1016/j.compositesa.2012.03.017.

[14] Boubaker B Ben, Haussy B, Ganghoffer JF. Consideration of the yarn-yarn interactions in meso/macro discrete model of fabric. Part II: Woven fabric under uniaxial and biaxial extension. Mech Res Commun 2007;34:371-8. doi:10.1016/j.mechrescom.2007.02.002.

[15] Boubaker B Ben, Haussy B, Ganghoffer JF. Mesoscopic fabric models using a discrete massspring approach: Yarn-yarn interactions analysis. J Mater Sci 2005;40:5925-32. doi:10.1007/s10853-005-5056-z.

[16] Boubaker B Ben, Haussy B, Ganghoffer JF. Discrete models of fabric accounting for yarn interactions: Simulations of uniaxial and biaxial behaviour. Rev Eur Des Elem 2005;14:65375. doi:10.3166/reef.14.653-675.

[17] Boubaker B Ben, Haussy B, Ganghoffer JF. Consideration of the yarn-yarn interactions in meso/macro discrete model of fabric. Part I: Single yarn behaviour. Mech Res Commun 2007;34:359-70. doi:10.1016/j.mechrescom.2007.02.003.

[18] Hivet G, Boisse P. Consistent mesoscopic mechanical behaviour model for woven composite reinforcements in biaxial tension. Compos Part B Eng 2008;39:345-61. doi:10.1016/j.compositesb.2007.01.011.

[19] Cox BN, Carter WC, Fleck N a. A binary model of textile composites-I. Formulation. Acta Metall Mater 1994;42:3463-79. doi:10.1016/0956-7151(94)90479-0.

[20] Xu J, Cox BN, McGlockton MA, Carter WC. A binary model of textile composites-II. The elastic regime. Acta Metall Mater 1995;43:3511-24. doi:10.1016/0956-7151(95)00057-3.

[21] McGlockton MA, Cox BN, McMeeking RM. A Binary Model of textile composites: III high failure strain and work of fracture in 3D weaves. J Mech Phys Solids 2003;51:1573-600. doi:10.1016/S0022-5096(03)00038-3.

[22] Yang Q, Cox B. Spatially Averaged Local Strains in Textile Composites Via the Binary Model Formulation. J Eng Mater Technol 2003;125:418. doi:10.1115/1.1605117.

[23] Flores S, Evans AG, Zok FW, Genet M, Cox B, Marshall D, et al. Treating matrix nonlinearity in the binary model formulation for 3D ceramic composite structures. Compos Part A Appl Sci Manuf 2010;41:222-9. doi:10.1016/j.compositesa.2009.10.020.

[24] Scida D, Aboura Z, Benzeggagh ML, Bocherens E. A micromechanics model for 3D elasticity and failure of woven-fibre composite materials. Compos Sci Technol 1999;59:505-17. doi:10.1016/S0266-3538(98)00096-7.

[25] Naik NK, Tiwari SI, Kumar RS. An analytical model for compressive strength of plain weave fabric composites. Compos Sci Technol 2003;63:609-25. doi:10.1016/S0266-3538(02)001987.

[26] Escárpita DAA. Experimental Investigation of Textile Composites Strength Subject to Biaxial Tensile Loads. Instituto Tecnologico y de Estudios Superiores de Monterrey, 2011.

[27] Chaouachi F, Rahali Y, Ganghoffer JF. A micromechanical model of woven structures accounting for yarn-yarn contact based on Hertz theory and energy minimization. Compos Part 
B Eng 2014;66:368-80. doi:10.1016/j.compositesb.2014.05.027.

[28] Jones RM. Mechanics of composite materials. second edi. Taylor \& Francis; 1999.

[29] Bai JB, Xiong JJ, Shenoi RA, Zhu YT. Analytical solutions for predicting tensile and in-plane shear strengths of triaxial weave fabric composites. Int J Solids Struct 2017;120:1339-51. doi:10.1016/j.ijsolstr.2017.05.002.

[30] Wang Z, Bai J, Sobey AJ, Xiong J, Shenoi RA. Optimal design of triaxial weave fabric composites under tension. Compos Struct 2018;201:616-24. doi:https://doi.org/10.1016/j.compstruct.2018.06.090.

[31] Gross D, Hauger W, Schröder J, Wall WA, Bonet J. Bending and Tension/Compression. Engineering Mechanics 2 - Mechanics of Materials, Springer Berlin Heidelberg; 2011, p. 171.

[32] Daniel IM, Ishai O. Longitudinal Tension - Failure Mechanisms and Strength. Engineering Mechanics of Composite Materials (Second edition), New York: Oxford University Press; 2006, p. 98-9.

[33] Yetman JE, Sobey AJ, Blake JIR, Shenoi RA. Mechanical and fracture properties of glass vinylester interfaces. Compos Part B Eng 2017;130:38-45. doi:10.1016/j.compositesb.2017.07.011.

[34] Ansari R, Hassanzadeh-Aghdam MK, Darvizeh A. On elastic modulus and biaxial initial yield surface of carbon nanotube-reinforced aluminum nanocomposites. Mech Mater 2016;101:1426. doi:10.1016/j.mechmat.2016.07.008.

[35] Hasanzadeh M, Ansari R, Hassanzadeh-Aghdam MK. Micromechanical elastoplastic analysis of randomly oriented nonstraight carbon nanotube-reinforced polymer nanocomposites. Mech Adv Mater Struct 2018:1-11. doi:10.1080/15376494.2018.1444227.

[36] Tsai SW, Wu EM. A general theory of strength for anisotropic materials. J Compos Mater 1971;5:58-80.

[37] Kollegal MG, Sridharan S. A simplified model for plain woven fabrics. J Compos Mater 2000;34:1756-86.

[38] Liu Z, Zhu C, Zhu P, Chen W. Reliability-based design optimization of composite battery box based on modified particle swarm optimization algorithm. Compos Struct 2018. doi:10.1016/j.compstruct.2018.07.053.

[39] Barthélémy H. Hydrogen storage - Industrial prospectives. Int J Hydrogen Energy 2012;37:17364-72. doi:10.1016/j.ijhydene.2012.04.121. 